\title{
Magnetic Nanostructures Patterned by Focused Ion Beam in an ultrathin $\mathbf{P t} / \mathbf{C o} / \mathbf{P t}$ film
}

\author{
R. Hyndman, A. Mougin, V. Repain, J. Ferré, J.P. Jamet, J. Gierak*, D. Mailly*, C. Chappert**, V. Mathet**, \\ P.Warin***, J.N. Chapman***
}

\begin{abstract}
Laboratoire de Physique des Solides, UMR CNRS 8502, Univ. Paris-Sud, 91405 Orsay, France
*Laboratoire de Physique et de Nanostructures, Route de Nozay, 91460 Marcoussis, France

**Institut d'Electronique Fondamentale, UMR CNRS 8622, Univ. Paris-Sud, 91405 Orsay, France

***Department of Physics and Astronomy, Univ. of Glasgow, Glasgow G12 8QQ, U.K
\end{abstract}

The magnetic properties of $\mathrm{Co} / \mathrm{Pt}$ films can be modified and even controlled by ion beam irradiation. For example, the coercivity and magnetic anisotropy of a $1.4 \mathrm{~nm}$ thick Co layer are reduced with increasing the $\mathrm{Ga}^{+}$ ion dose. Images of the demagnetized states and remanent hysteresis loops are obtained for arrays of dots or tracks patterned by the focused ion beam technique. $20 \mathrm{~nm}$ wide sharp non-magnetic irradiated lines separate the magnetic dots or the tracks, the size of the dots or the width of the tracks varying from $1.8 \mu \mathrm{m}$ down to $70 \mathrm{~nm}$. The efficiency of the dipolar interaction is studied, and the use of such dot arrays for magnetic or magneto-optical recording discussed.

Key words: ion beam patterning, magnetic dot arrays, magneto-optical microscopy, magneto-optical recording

\section{Introduction}

One approach to designing potentially new, ultra-high density magnetic or magneto-optic storage media is based on arrays of sub-micron elements patterned on ferromagnetic thin films. Etching or patterning of metallic multilayers by a Ga ${ }^{+}$Focused Ion Beam (FIB) provides an elegant and versatile technique to design well defined magnetic patterns ${ }^{1-4)}$. In this article we concentrate our studies on a $\mathrm{Pt} / \mathrm{Co} / \mathrm{Pt}$ single magnetic layer film structure with perpendicular anisotropy. The film is prepared by sputtering onto an $\mathrm{Al}_{2} \mathrm{O}_{3}$ (0001) single crystal substrate. The structural properties are optimized for substrate temperatures near $600^{\circ} \mathrm{C}\left(300^{\circ} \mathrm{C}\right)$ during $\mathrm{Pt}(\mathrm{Co})$ deposition. It has already been shown that the magnetic state of a sample irradiated with low $\mathrm{He}^{+}$or $\mathrm{Ga}^{+}$ion doses can be modified and even controlled in a simple way, ${ }^{5}$. For example, by uniform $\mathrm{He}^{+}$irradiation, the perpendicular coercivity of a $\mathrm{Pt} / \mathrm{Co}(1.4 \mathrm{~nm}) / \mathrm{Pt}$ film is reduced with increased dose, becoming paramagnetic at $2 \times 10^{16} \mathrm{He}^{+}$ions $/ \mathrm{cm}^{2}$, without modifying the planarity of the film ${ }^{5)}$. The situation is different for a $[\mathrm{Pt} / \mathrm{Co}]_{5} / \mathrm{Pt}$ multilayer structure, where weak $\mathrm{He}^{+}$irradiation switches the magnetic anisotropy from in-plane to perpendicular ${ }^{5}$. Similar effects are provided by uniform $\mathrm{Ga}^{*}$ irradiation, except that these heavy ions are about ten times more efficient than $\mathrm{He}^{+}$. They create relatively more damage inside the $\mathrm{Co} / \mathrm{Pt}$ film structure and increase the film roughness ${ }^{6}$.

In this paper we report first on the main morphology and magnetic changes in $\mathrm{Co} / \mathrm{Pt}$ film structures under quasi-uniform irradiation by $\mathrm{Ga}^{+}$FIB. The quasi-static magnetic properties of arrays of $\mathrm{Pt} / \mathrm{Co} / \mathrm{Pt}$ dots or tracks, obtained by patterning the film with identical sharp FIB irradiated lines, are studied by high resolution magnetooptical microscopy. The role of dipolar interactions is discussed. Finally, the change in magnetic properties of these arrays on reducing the size of the magnetic elements is investigated and discussed in the framework of new magneto-optical recording media.

\section{Quasi-uniform $\mathrm{Ga}^{+}$irradiation}

A quasi-uniform ion irradiation is obtained by scanning the FIB spot along two orthogonal directions in the film plane. The profile of the FIB spot is close to being Gaussian with a diameter at half height estimated here at $10 \mathrm{~nm}$. The distances between two FIB spot centers and between the axes of adjacent irradiated lines are also 10 $\mathrm{nm}$. Thus, a quasi-homogeneous surface $\mathrm{Ga}^{+}$ion dose D can be defined.

For $\operatorname{Pt}(3.4 \mathrm{~nm}) / \mathrm{Co}(1.4 \mathrm{~nm}) / \mathrm{Pt}(4.5 \mathrm{~nm}) /$ sapphire samples, we find that at low doses the etching depth varies linearly with D. Estimates from TRIM simulations supported by direct measurements show that a dose of $10^{15}$ ions $/ \mathrm{cm}^{2}$ removes $1-3 \mathrm{~nm}$ of material ${ }^{6,77}$. The $\mathrm{Ga}^{+}$ion implants to an average depth of $6 \mathrm{~nm}$ inside the ultrathin film structure. 


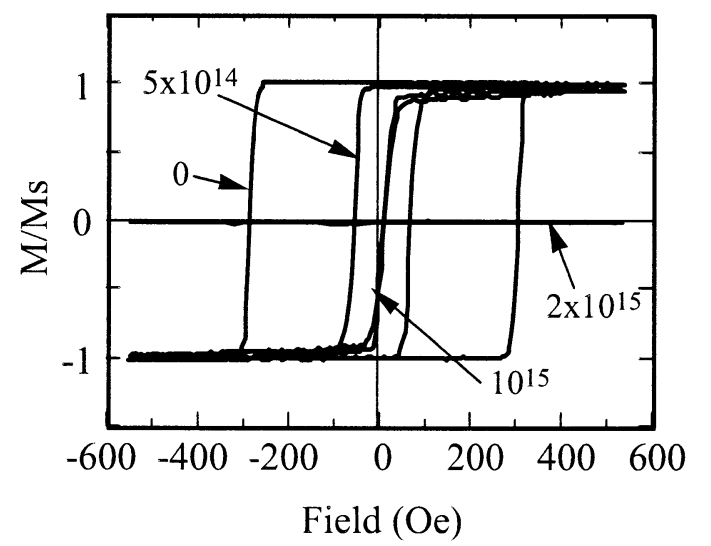

Fig. 1 Renormalized Kerr hysteresis loops of the $\mathrm{Co}(1.4 \mathrm{~nm})$ layer for several quasi-homogeneous irradiations with doses indicated in $\mathrm{Ga}^{+}$ions $/ \mathrm{cm}^{2}$.

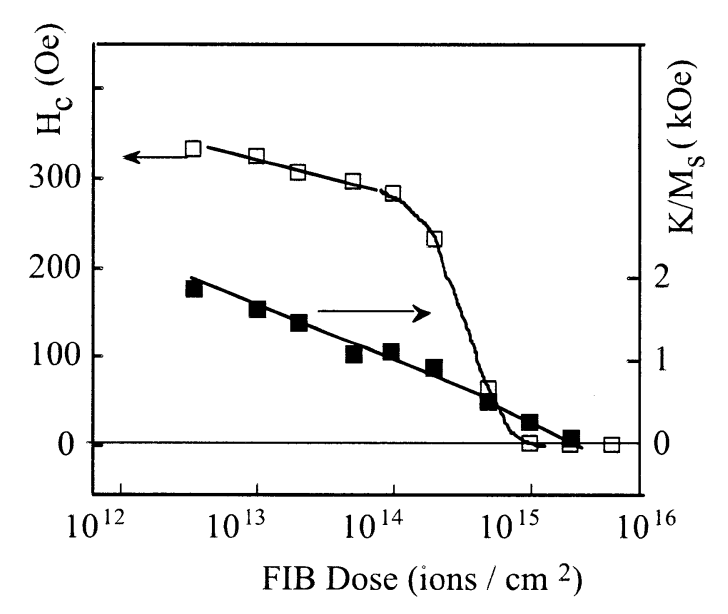

Fig. 2 Variation of the coercive field (open squares left scale) and anisotropy (full squares - right scale) of the $\operatorname{Co}(1.4 \mathrm{~nm})$ layer with the FIB.

The out-of-plane coercivity and anisotropy (Fig. 1, 2) and Curie temperature are reduced under irradiation but the polar hysteresis loop remains square. The coercivity drops rapidly for $\mathrm{D}>2 \times 10^{14}$ ions $/ \mathrm{cm}^{2}$ and vanishes for $\mathrm{D}$ $=10^{15}$ ions $/ \mathrm{cm}^{2}$. Then, the Co layer becomes paramagnetic for $\mathrm{D} \geq 2 \times 10^{15}$ ions $/ \mathrm{cm}^{2}$, even though the etching of the Pt overlayer is only $2.6 \mathrm{~nm}$ and the Co layer remains intact. This means that, as for $\mathrm{He}^{+}$ion irradiation ${ }^{8)}$, the main role of the $\mathrm{Ga}^{+}$irradiation is to modify the local arrangement of the Co and $\mathrm{Pt}$ ions in the vicinity of the Co-Pt interfaces. Since anisotropy is very sensitive to the nature of the interface, the coercivity is modified accordingly 6 ).

In comparison, for the $[\mathrm{Pt} / \mathrm{Co}]_{5} / \mathrm{Pt}$ multilayer, the outof-plane anisotropy persists only at very low doses, up to $7 \times 10^{12}$ ions $/ \mathrm{cm}^{2}$, then falls in-plane at higher doses ${ }^{7}$.

\section{Arrays of magnetic dots or tracks}

We have already reported on the magnetic behavior of $\mathrm{Pt} / \mathrm{Co}(1.4 \mathrm{~nm}) / \mathrm{Pt}$ square $\left(1 \mu \mathrm{m} \times 1^{1} \mu \mathrm{m}\right)$ dot arrays patterned by FIB at relatively high dose ${ }^{1)}$. In that case the non-magnetic width of the irradiated lines separating the magnetic dots was estimated to be $30 \mathrm{~nm}$. Here, we present data for dot and track arrays patterned with FIB irradiated lines etched at a smaller dose $\mathrm{D}=5 \times 10^{15}$ ions $/ \mathrm{cm}^{2}$. As a consequence, the width of the central nonmagnetic region of the irradiated lines is smaller and estimated at $20 \mathrm{~nm}$. Hence, in terms of magnetic recording, it is possible to enhance the bit density, but unfortunately the dipolar coupling between dots becomes larger. Polar magneto-optical images of parts of the square dot arrays in their demagnetized state and with various square dot size $\mathrm{d}$, ranging from 1800 to $70 \mathrm{~nm}$, are presented on figure 3 .

One expects that, at our long time observation scale, even the smallest dots $(70 \mathrm{~nm} \times 70 \mathrm{~nm} \times 1.4 \mathrm{~nm})$ must be single ferromagnetic domains. Superparamagnetism is only expected for particles with smaller volume. As expected, and in accord with previous simulations ${ }^{1)}$, dipolar interaction through the narrow irradiated nonmagnetic lines favors checkerboard magnetic configurations in the demagnetized state of the arrays. Since an increase of D enhances the Kerr magneto-optical effect $^{6)}$, the local magnetic region surrounding the FIB lines appears whiter (Fig. 3a,b) and is clearly visible in

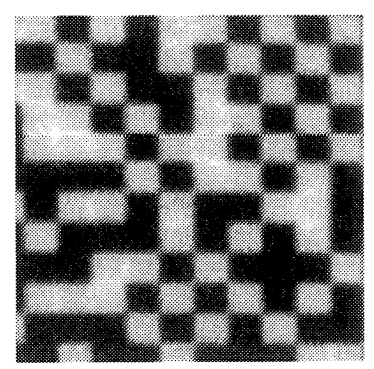

(a)

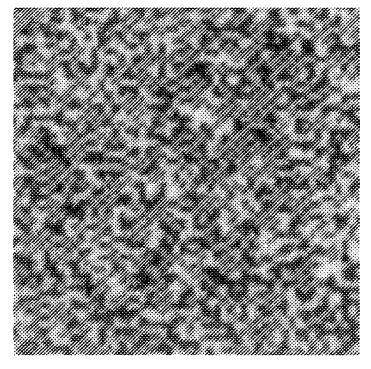

(c)

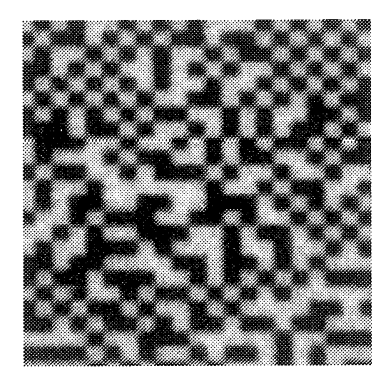

(b)

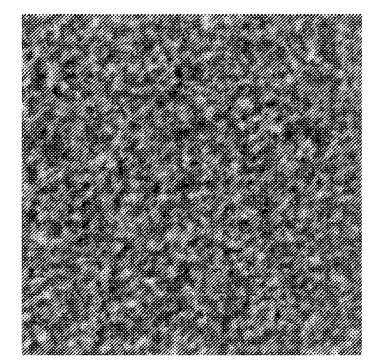

(d)
Fig. 3 Magneto-optical images $(18 \mu \mathrm{m} \times 18 \mu \mathrm{m})$ of the central part of the demagnetized dot arrays with dot sizes: (a) $1800 \mathrm{~nm}$, (b) $900 \mathrm{~nm}$, (c) $340 \mathrm{~nm}$, (d) $70 \mathrm{~nm}$ 
spite of its narrowness. Whereas individual dots can be clearly identified for $\mathrm{d} \geq 340 \mathrm{~nm}$, the limited optical resolution of magneto-optical microscopy only permits a global overview of the magnetization state for arrays of smaller dots.

The corresponding remanent hysteresis loops for arrays with different dot dimension $\mathrm{d}$, are shown on Fig. 4. The data are extracted from the magnitude of the remanent magneto-optical Kerr effect measured in zero field after applying a field $\mathrm{H}$ for $5 \mathrm{~s}$. As the size of the dots is reduced, the coercive field of the arrays $\left(\mathrm{H}_{\mathrm{C} 0}\right)$ as a whole increases slightly compared to the virgin film. Then, for $\mathrm{d}$ $<340 \mathrm{~nm}$, it decreases quite rapidly.

In spite of the dipolar effect, the first increase of $\mathrm{H}_{\mathrm{C} 0}$ with $\mathrm{D}$ is related to the low probability of nucleation inside small dots ${ }^{9)}$ or at their boundaries ${ }^{1)}$. In previous experiments ${ }^{1)}$, irradiation tails from the FIB spot provided low field nucleation areas around the borders of each dot. However, as FIB resolution is increased here, which means sharper irradiated lines, the irradiation tails do not spread enough to stabilize a reversed domain state and initiate a nucleation state at low field.

The reduction of the coercive field of dot arrays with smaller periods is certainly due to the increasing importance of the irradiation inside dots. As FIB irradiated lines become closer, the region between these lines can be partially irradiated by the tails of the FIB dose distribution $^{10)}$. As we have already stated, the FIB lines are quite sharp and from TRIM simulations we find that this effect is only significant for arrays with the smallest dots $(\mathrm{d}<200 \mathrm{~nm})$. On the other hand, at smaller period and dot size $d$, each dot experiences a larger dipolar field, which explains why the magnetization in many dots begins to switch easily at a small field (Fig. 4). Within a simple giant spin model, the dipolar field experienced at the dot center scales as $1 / \mathrm{d}$, taking into account the variation of the dot volume and spacing. For the smallest

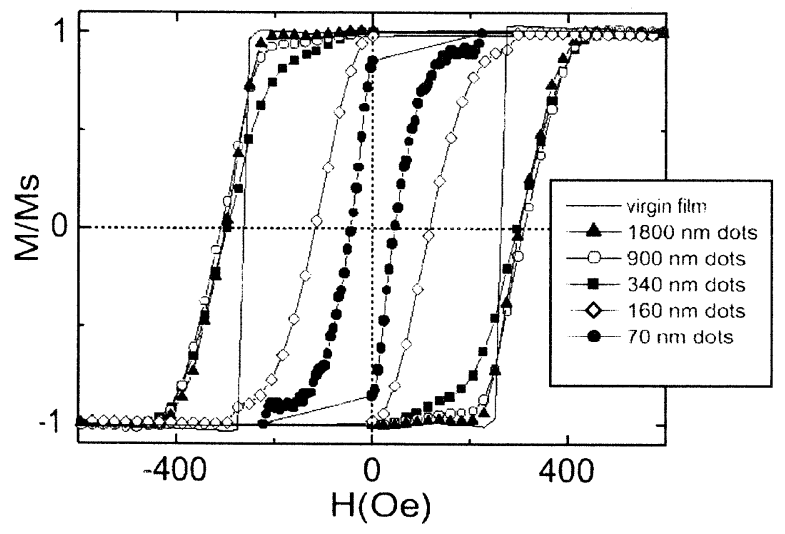

Fig. 4 Renormalized Kerr hysteresis loops for

\section{different dot arrays}

size dot $(\mathrm{d}<100 \mathrm{~nm})$ arrays, the dramatic reduction of coercivity is probably due to a combination of efficient dipolar interactions and partial irradiation of the interior of the dots itself.

Demagnetized states of several track arrays with different track widths are presented in Fig. 5. Again, the dipolar coupling favors an anti-parallel orientation of the magnetization in neighbouring tracks. The track arrays are separated from the virgin film by a heavily irradiated FIB line (not seen in the figure since Fig. 5 shows only the central part of the arrays) following the perimeter of the arrays. The magnetization reversal in tracks is initiated at their end, along the heavy FIB line surrounding the square array, and proceeds by domain wall propagation along their length. Thanks to our good optical resolution, the orientation of track may be visualized even for the $d=$ $160 \mathrm{~nm}$ array (Fig. 5d).

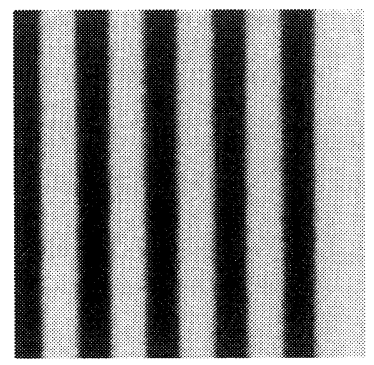

(e)

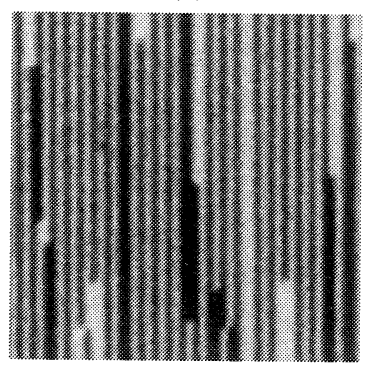

(c)

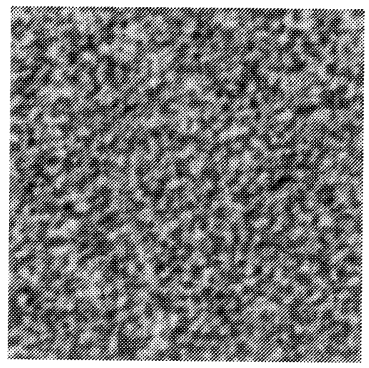

(e)

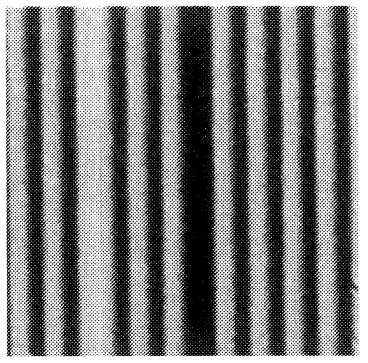

(b)

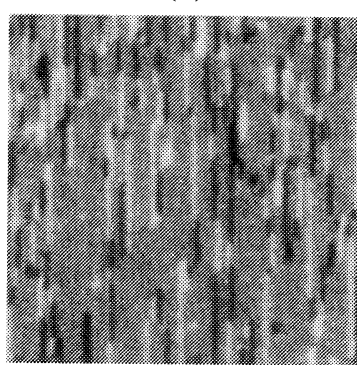

(d)
Fig. 5 Magneto-optical images of the central part (18 $\mu \mathrm{m} \times 18 \mu \mathrm{m})$ of the demagnetized state of the arrays of magnetic tracks with width: (a) $1800 \mathrm{~nm}$, (b) $900 \mathrm{~nm}$, (c) $340 \mathrm{~nm}$, (d) $160 \mathrm{~nm}$, (e) $70 \mathrm{~nm}$. 
The remanent hysteresis loops of these track arrays are shown on Fig. 6. The variation of the loop shape and coercivity with the $\mathrm{Ga}^{+}$ion dose is comparable to that found for the dot arrays (Fig. 4). However, for low d values, the coercivity is higher for the track arrays. This is expected since irradiation and dipolar effects are less efficient in track arrays.

In previous work ${ }^{11)}$, it was shown that it is possible to retain some exchange interaction between dots at the lower doses. Then, the ratio between the exchange interaction and the dipolar coupling between dots can be modified by changing the irradiation dose in the FIB lines. As a consequence, the details of the magnetic

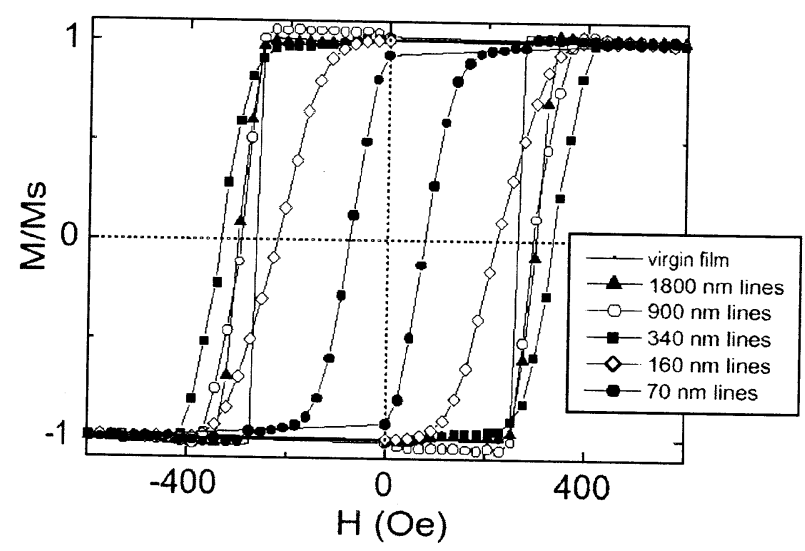

Fig. 6 Renormalized Kerr hysteresis loops for different track arrays

behavior of a granular magnetic recording media, where both dipolar and intergrain exchange interaction coexist together, may be revealed in our model system.

From this study we have stressed that it is possible to stabilize magnetized configurations (Figs. 4, 6) in a $\mathrm{Pt} / \mathrm{Co}(1.4 \mathrm{~nm}) / \mathrm{Pt}$ dot array with dot size as small as $70 \mathrm{~nm}$, separated by $20 \mathrm{~nm}$ wide non-magnetic FIB lines. This result pushes down a little the size limits as compared to previous writing demonstrations in FIB patterned arrays in the $\mathrm{Co}_{70} \mathrm{Cr}_{18} \mathrm{Pt}_{12}$ recording media ${ }^{3)}$. In order to increase the magnetic stability without losing information density one has to reduce significantly the dipolar field effect by increasing the distance between dots, and reducing simultaneously the dimension of the dots by nearly the same amount.

\section{Reference}

[1] T. Aign, J. Ferré, P. Meyer, S. Lemerle, J.P. Jamet, V. Mathet, C. Chappert, J. Gierak, C. Vieu, F. Rousseaux, H. Launois and H. Bernas, Phys. Rev. Lett. 81, 5656 (1998)

[2] J.P. Jamet, T. Aign, P. Meyer, S. Lemerle, J. Ferré, J. Gierak, C. Vieu, H. Bernas, T. Devolder, V. Mathet, C. Chappert, E. Cambril, D. Decanini and H. Launois, J. Magn. Soc. Jpn 23, S1, 71 (1999)

[3] J. Lohau, A. Moser, C.T. Rettner, M.E. Best and B.D. Terris, Appl. Phys. Lett. 78, 990 (2001)

[4] P. Warin, R. Hyndman, J. Gierak, J.N. Chapman, J. Ferré, J .P. Jamet, V. Mathet and C. Chappert, J. Appl. Phys. 90, 3850 (2001)

[5] C. Chappert, H. Bernas, J. Ferré, V. Kottler, J.P. Jamet, Y. Chen, E. Cambril, T. Devolder, F. Rousseaux, V. Mathet and H. Launois, Science 280, 1919 (1998)

[6] C. Vieu, J. Gierak, H. Launois, T. Aign, P. Meyer, J.P. Jamet, J. Ferré, C. Chappert, T. Devolder, V. Mathet and H . Bernas, J. Appl. Phys. 91, 3103 (2001)

[7] R. Hyndman, P. Warin, J. Gierak, J. Ferré, J.N. Chapman, J .P. Jamet, V. Mathet and C. Chappert, J. Appl. Phys. 90, 3843 (2001)

[8] T. Devolder, Phys. Rev. B62, 5794 (2000)

[9] J.P. Jamet, S. Lemerle, P. Meyer, J. Ferré, B. Bartenlian, N. Bardou, C. Chappert, P. Veillet, F. Rousseaux, D. Decanini and H. Launois, Phys. Rev. B57, 14320 (1998)

[10] J.P. Jamet, J. Ferré, P. Meyer, J. Gierak, C. Vieu, F. Rousseaux, C. Chappert and V. Mathet, IEEE Trans. Magn. 37, 2120 (2001)

[11] R. Hyndman, P. Meyer, J. Ferré, J.P. Jamet, T. Devolder, V. Mathet, C. Chappert and J. Gierak, J. Magn. Soc. Jpn 25, 198 (2001)

Recerived April 29, 2002; Accepted July 5, 2002 